\title{
Preditores de recorrência de lesões autoprovocadas e de óbitos por suicídio em um
}

\section{estado brasileiro}

\author{
Predictors of recurrence of self-harm and suicide deaths in a Brazilian state \\ Predictores de recurrencia de autolesiones y muertes por suicidio en un estado brasileño
}

Recebido: 19/01/2021 | Revisado: 23/01/2021 | Aceito: 26/01/2021 | Publicado: 03/02/2021

\author{
Nágella Thaysa Bier de Sousa \\ ORCID: https://orcid.org/0000-0001-8820-5091 \\ Universidade de São Paulo, Brasil \\ E-mail: nagella@usp.br \\ Letícia Oliveira Othon Teixeira \\ ORCID: https://orcid.org/0000-0003-1051-4622 \\ Universidade de São Paulo, Brasil \\ E-mail: leticiaothon@usp.br \\ Kelly Graziani Giacchero Vedana \\ ORCID: http://orcid.org/0000-0001-7363-2429 \\ Universidade de São Paulo, Brasil \\ E-mail: kellygiacchero@eerp.usp.br \\ Adriana Inocenti Miasso \\ ORCID: https://orcid.org/0000-0003-1726-7169 \\ Universidade de São Paulo, Brasil \\ E-mail: amiasso@eerp.usp.br
}

\begin{abstract}
Resumo
Objetivos: caracterizar as lesões autoprovocadas e investigar preditores sociodemográficos e clínicos para recorrência de lesões autoprovocadas e para óbitos por suicídio no Estado do Mato Grosso Métodos: Trata-se de estudo transversal, analítico, de abordagem quantitativa, que incluiu todos os casos de lesões autoprovocadas e óbitos por suicídio registrados em sistemas de informação em saúde. Foram desenvolvidos modelos de regressão logística para analisar os preditores dos desfechos de interesse. Resultados: Os resultados revelaram 1507 notificações de lesões autoprovocadas, sendo que 1443 pessoas tiveram apenas uma lesão autoprovocada e 29 pessoas com recorrência de tais lesões. Das 1472 pessoas com lesão autoprovocada na primeira notificação, 424 foram a óbito. Houve maior chance de recorrência de lesão autoprovocada em pessoas com idade de 10 a 19 anos (OR 2,28, IC 95\% 1,09 - 4,79), com algum tipo de deficiência ou transtorno (OR 1,85, IC 95\% 1,13 - 3,03). Identificou-se menor chance de óbito por suicídio em pessoas com idade de 10 a 19 anos (OR 0,06, IC 95\% 0,02 - 0,17), e de 20 a 59 anos (OR 0,23, IC 95\% 0,10 - 0,54), com até oito anos de escolaridade (OR 0,23, IC 95\% 0,15 - 0,36), mulheres (OR 0,21, IC 95\% 0,13 - 0,32) e por autointoxicação intencional por medicamento (OR 0,17, IC 95\% 0,08 - 0,38). Conclusão: Este estudo fornece subsídios para estratégias de vigilância à saúde da população para prevenção de suicídio, além de indicar a necessidade de ações que promovam notificação adequada pelos instrumentos disponibilizados nos serviços de saúde.
\end{abstract}

Palavras-chave: Tentativa de suicídio; Suicídio; Saúde mental; Comportamento autodestrutivo.

\begin{abstract}
Objectives: to characterize self-harm and to investigate sociodemographic and clinical predictors for recurrence of selfharm and death by suicide in the State of Mato Grosso Methods: This is a cross-sectional, analytical study with a quantitative approach, which included all cases of self-harm. and suicide deaths recorded in health information systems. Logistic regression models were developed to analyze the predictors of the outcomes of interest. Results: The results revealed 1507 notifications of self-harm, with 1443 people having only one self-harm and 29 people with recurrence of such injuries. Of the 1472 people with self-harm in the first notification, 424 died. There was a greater chance of recurrence of self-harm in people aged 10 to 19 years (OR 2.28, 95\% CI 1.09 - 4.79), with some type of disability or disorder (OR 1.85, 95 CI \% 1.13 - 3.03). A lower chance of death by suicide was identified in people aged 10 to 19 years (OR 0.06, 95\% CI 0.02 - 0.17), and from 20 to 59 years old (OR $0.23,95$ CI \% 0.10 - 0.54), with up to eight years of schooling (OR 0.23, 95\% CI 0.15 - 0.36), women (OR 0.21, 95\% CI 0.13 - 0, 32) and intentional autointoxication by medication (OR 0.17, 95\% CI 0.08 - 0.38). Conclusion: This study provides subsidies for population health surveillance strategies for suicide prevention, in addition to indicating the need for actions that promote adequate notification by the instruments available in health services.
\end{abstract}

Keywords: Suicide attempted; Suicide; Mental health; Self-injurious behavior. 


\section{Resumen}

Objetivos: caracterizar la autolesión e investigar predictores sociodemográficos y clínicos de recurrencia de autolesión y muerte por suicidio en el estado de Mato Grosso. Métodos: Se trata de un estudio analítico transversal con enfoque cuantitativo, que incluyó todos los casos de autolesión. y muertes por suicidio registradas en los sistemas de información de salud. Se desarrollaron modelos de regresión logística para analizar los predictores de los resultados de interés. Resultados: Los resultados revelaron 1507 notificaciones de autolesiones, 1443 personas que sufrieron una sola autolesión y 29 personas con recurrencia de dichas lesiones. De las 1472 personas que se autolesionaron en la primera notificación, 424 murieron. Hubo una mayor probabilidad de recurrencia de autolesiones en personas de 10 a 19 años (OR 2,28; IC del 95\%: 1,09 - 4,79), con algún tipo de discapacidad o trastorno (OR 1,85, IC del 95\%). \% 1,13 - 3,03). Se identificó una menor probabilidad de muerte por suicidio en personas de 10 a 19 años (OR 0,06; IC del 95\%: 0,02 - 0,17) y de 20 a 59 años (OR 0,23; IC del 95\% \% 0,10 - 0,54), con hasta ocho años de escolaridad (OR 0,23, IC del 95\%: 0,15 0,36), mujeres (OR 0,21, IC del 95\% 0,13 - 0,32) y autointoxicación intencional por medicación (OR 0,17; IC del 95\%: 0,08 - 0,38). Conclusión: Este estudio otorga subsidios a las estrategias de vigilancia de la salud de la población para la prevención del suicidio, además de señalar la necesidad de acciones que promuevan la adecuada notificación por los instrumentos disponibles en los servicios de salud.

Palabras clave: Intento de suicidio; Suicidio; Salud mental; Conducta autodestructiva.

\section{Introdução}

A lesão autoprovocada é um tipo de violência autoinfligida, podendo ser subdividida em comportamento suicida (pensamentos, tentativas e consumação) e autoagressão (inclui atos de automutilação) (World Health Organization [WHO], 2002, 2014) consistindo em grave problema de saúde pública.

No Brasil, entre os anos 2011 e 2014, houve 67.388 notificações de lesões autoprovocadas realizadas pelos serviços de saúde, correspondendo a 14\% de todos os registros de violências (Bahia, Avanci, Pinto, \& Minayo, 2020). Em relação ao comportamento suicida, destaca-se que cerca de 800 mil pessoas morrem por suicídio todos os anos, o que resulta em uma morte por suicídio a cada 40 segundos, sendo a segunda causa de morte de jovens com idade de 15 a 29 anos e a terceira causa principal entre pessoas de 15 a 39 anos (WHO, 2014).

A literatura aponta vários fatores relacionados ao suicídio, com destaque para faixa etária, sexo, localizações geográficas (Cicogna, Hillesheim, \& Hallal, 2019), raça (Machado \& Santos, 2015), nível de escolaridade, estado civil, renda, ocupação, orientação sexual e religião (Conejero, Castroman-Lopez, Giner, \& Baca-Garcia, 2016). Em relação aos fatores de risco para tentativas de suicídio, destacam-se idade jovem, sexo feminino, ser estudante com ensino médio completo, além da presença de transtornos psiquiátricos e de histórico pessoal e familiar de tentativa de suicídio (Omezzine, 2019). Estudo de Parra-Uribe, Blasco-Fontecilla, Garcia-Parés, Martínez-Naval, Valero-Coppin, Cebrià-Meca, Oquendo e Palao-Vidal (2017) identificou, na amostra analisada, que os riscos de recorrência da tentativa de suicídio estiveram relacionados ao uso de álcool, transtornos de personalidade e idade de 30 a 39 anos.

Finkelstein, Macdonald, Hollands, Sivilotti, Hutson, Mamdani, Koren e Juurlink (2015) identificaram a história anterior de tentativa de suicídio como maior preditor de comportamento suicida futuro, incluindo ideação, tentativas e óbito por suicídio. Após uma tentativa de suicídio não fatal, a pessoa usualmente opta progressivamente por meios mais letais em tentativas futuras (Chen, Liao, Lee, Wu, Lin, \& Chen, 2016). Portanto, uma forma de prevenir o óbito por suicídio consiste em identificar e intervir nos fatores de risco para tentativa de suicídio (Omezzine, 2019).

Estudos internacionais revelam aumento da mortalidade por suicídio em vários países (Fond, Llorca, Boucekine, Zendjidjian, Brunel, Lancon, Auquier, \& Boyer, 2016; India State-Level Disease Burden Initiative Suicide Collaborators, 2018). Sinalizam que a mortalidade por suicídio é maior em homens do que em mulheres (Azcárate-Juménez, Goñi, GoñiSarriés, Reula-Montes, Fernández-Portilla, \& Elorza-Pardo, 2019; Haghparast-Bidgoli, Rinaldi, Shahnavazi, Bouraghi, \& Kiadaliri, 2018; Naghavi, 2016) e que as mulheres utilizam métodos de menor letalidade (Azcárate-Juménez et al., 2019). De acordo com os estudos, um dos métodos mais utilizados por pessoas do sexo feminino na tentativa de suicídio é a intoxicação exógena por medicamento (Azcárate-Juménez et al., 2019; Bochner \& Freire , 2018; Ferreira, Vedana, Amaral, Pereira, 
Zanetti, Miasso, \& Borges, 2019; Moreira, Martins, Gubert, \& Sousa, 2015; Omezzine, 2019; Wieczorkievicz, Mazon, Raissa, Maia, \& Siebeneichler, 2016) e os meios mais frequentes utilizados por pessoas do sexo masculino são enforcamento, precipitação em altura e armas de fogo (Azcárate, Peinado, Blanco, Goñi, Cuesta, Prandini, \& López-Goñi, 2015).

No Brasil, em 2016, o suicídio foi a terceira maior causa de morte no grupo etário de cinco a 29 anos, em ambos os sexos, apresentando taxas mais altas e crescentes no sexo masculino (Brasil, 2019). Estudo desenvolvido no período de 2000 a 2012 revelou que as tendências de mortalidade por suicídio divergem entre as regiões brasileiras apresentando índices de suicídio maiores nas regiões Sul e Centro-Oeste quando comparadas às regiões Norte, Nordeste e Sudeste (Machado \& Santos, 2015).

Estudo que descreveu a mortalidade por suicídio no Estado de Mato Grosso, no Brasil, no período de 1996 a 2015 , revelou média de 6,8 óbitos/100.000 habitantes, sendo tal valor superior ao Brasil que apresenta média de 5,7 óbitos/100.000 habitantes (Oliveira \& Benedetti, 2018).

Considerando a alta taxa de mortalidade por suicídio no Estado do Mato Grosso, a carência de estudos relacionados às lesões autoprovocadas (Vieira, Santana, \& Suchara, 2015) e informações pouco exploradas sobre o suicídio no referido Estado (Oliveira \& Benedetti, 2018), faz-se relevante conhecer o perfil sociodemográfico e clínico atualizado da ocorrência e recorrência de lesões autoprovocadas e de óbitos por suicídio visando à elaboração e implementação de programas, ações e políticas públicas voltadas para prevenção do suicídio.

Nessa direção, esse estudo teve como objetivo caracterizar as lesões autoprovocadas e investigar preditores sociodemográficos e clínicos para recorrência de lesões autoprovocadas e para óbitos por suicídio no Estado do Mato Grosso.

\section{Metodologia}

\subsection{Tipo de estudo e local}

Trata-se de estudo de abordagem quantitativa. Nos métodos quantitativos, faz-se a coleta de dados quantitativos ou numéricos por meio do uso de medições de grandezas e obtém-se por meio da metrologia, números com suas respectivas unidades. Estes métodos geram conjuntos de dados a serem analisados por meio de técnicas matemáticas como porcentagens, estatísticas e probabilidades, métodos numéricos, métodos analíticos e geração de equações e/ou fórmulas matemáticas que se possam ser aplicados a algum processo (Pereira, Shitsuka, Parreira, \& Shitsuka, 2018).

Estudo transversal, do tipo analítico, realizado a partir de dados secundários dos bancos de dados epidemiológicos nacionais do Sistema de Informação de Agravos de Notificação e do Sistema de Informação sobre Mortalidade do Brasil, com enfoque no Estado do Mato Grosso. O Estado do Mato Grosso foi escolhido como local do estudo devido às taxas elevadas de suicídio na região centro-oeste do Brasil e, em particular, no referido estado (Calixto Filho \& Zerbini, 2016; Machado \& Santos, 2015; Oliveira \& Benedetti, 2018).

\subsection{Participantes}

Foram incluídos neste estudo os registros de casos de lesões autoprovocadas e os óbitos por suicídio no período de um de julho de 2015 a 31 de dezembro de 2017, em todos os municípios do Estado de Mato Grosso, Brasil. A escolha deste período justifica-se pelo fato de um dos instrumentos utilizados na pesquisa, a Ficha de Notificação/Investigação de Violência Interpessoal/Autoprovocada, ter sido modificado em junho de 2015.

Os critérios de exclusão do estudo foram: casos duplicados no banco de dados; casos que não caracterizavam lesão autoprovocada devido à incongruência de informações; casos com informações sobre lesões autoprovocadas e óbitos por suicídio referentes a crianças menores de 10 anos. 
Ao todo foram contabilizadas 1507 lesões autoprovocadas ocorridas no Estado do Mato Grosso e registrados nos bancos de dados epidemiológicos nacionais no período estudado, sendo que 1443 pessoas apresentaram lesão autoprovocada apenas uma vez e 29 mais de uma vez. Das 1472 pessoas com lesão autoprovocada na primeira notificação, 424 foram a óbito, resultando em 1048 pessoas para análise da recorrência de lesão autoprovocada.

\subsection{Variáveis do estudo}

Para este estudo foram definidas como variáveis dependentes a recorrência de lesão autoprovocada e óbito por suicídio. As variáveis independentes foram aquelas relacionadas ao perfil sociodemográfico e clínico, incluindo a autointoxicação intencional por medicamento (AIM). Todas as informações foram obtidas por meio dos registros da Ficha de Notificação/Investigação de Violência Interpessoal/Autoprovocada, Ficha de Notificação/Investigação de Intoxicação Exógena e da Declaração de Óbito.

Destaca-se que houve percentual elevado de informações classificadas como "ignorada" no banco de dados, sendo que essa opção corresponde a um dos quadrículos presentes nos campos das fichas de notificação avaliadas.

No que se refere à Ficha de Notificação/Investigação de Violência Interpessoal/Autoprovocada, foram obtidas informações referentes às seguintes variáveis: faixa etária; sexo; raça/cor; escolaridade; zona de ocorrência; local de ocorrência; situação conjugal; orientação sexual; se possui algum tipo de deficiência e/ou transtorno; tipo de deficiência e/ou transtorno; suspeita de uso de álcool. Neste instrumento foram considerados casos de lesões autoprovocadas aqueles que apresentavam o campo "lesão autoprovocada" assinalado. Para selecionar os casos de AIM, foi analisado se no item "meio de agressão" estava assinalada a opção "envenenamento/intoxicação".

Em relação à Ficha de Notificação/Investigação de Intoxicação Exógena foram obtidas informações referentes às seguintes variáveis: faixa etária; sexo; raça/cor; escolaridade; zona de ocorrência; local de ocorrência; situação no mercado de trabalho. Neste instrumento foram consideradas lesões autoprovocadas todos os casos referentes às fichas em que a “circunstância da exposição/contaminação" correspondia à "tentativa de suicídio". Para selecionar as AIM foi analisado se no item "grupo de agente tóxico" estava assinalada a opção "medicamento".

Quanto à Declaração de Óbito, foram obtidas informações referentes às seguintes variáveis: faixa etária; sexo; raça/cor; escolaridade; semestre da ocorrência; local de ocorrência; situação conjugal. Para identificação de óbitos por suicídio por AIM foram coletadas as informações contidas no campo "causa da morte" e realizada a classificação de acordo com a Classificação Estatística Internacional de Doenças e Problemas Relacionados com a Saúde, Décima Revisão (CID-10, World Health Organization, 2016). O suicídio não possui classificação específica na CID-10, no entanto, os fatores que envolvem as lesões autoprovocadas e o risco de suicídio são abordados em categorias diagnósticas que tratam de Lesões Autoprovocadas Intencionalmente (códigos CID-10: X60 a X69). As categorias diagnósticas que tratam de Lesões Autoprovocadas Intencionalmente por autointoxicações por medicamentos são X60, X61, X63 e X64.

\subsection{Análise dos dados}

Para a união das bases de dados, foi utilizado o método de linkage probabilístico, uma vez que não havia um campo identificador único entre as bases de dados. Esse método é baseado na utilização de combinações de campos comuns (no caso deste estudo, nome do paciente, nome da mãe, data de nascimento e sexo) presentes nas bases a serem relacionadas. Esses campos são utilizados conjuntamente para o cálculo de um escore, o qual traduz o grau de concordância entre os registros de cada link formado. Valores de escores limiares acima de 0,97 foram considerados pares verdadeiros. No caso de valores entre 0,70 e 0,97 optou-se por observar detalhadamente cada caso para, manualmente, classificar em par verdadeiro ou falso (Jaro, 1995; Camargo \& Coeli, 2000). 
A análise dos preditores de recorrências das lesões autoprovocadas, bem como dos óbitos por suicídio, foi realizada utilizando-se o software Statistical Analysis System (SAS), versão 9.2. Esses desfechos foram analisados por meio de modelo de regressão logística com efeitos aleatórios pertencentes à classe dos modelos Generalized Estimating Equations (GEE), escolhido por ter a possibilidade de uma mesma pessoa ter apresentado lesão autoprovocada mais de uma vez. Para todos os modelos de regressão logística foram calculados o Odds Ratio (OR) e seus respectivos intervalos de confiança de $95 \%$. Adotou-se um nível de significância de 5\% para todas as análises.

O estudo foi aprovado pelo Comitê de Ética em Pesquisa da Escola de Enfermagem de Ribeirão Preto - USP e seguiu as recomendações da Resolução n ${ }^{\circ}$ 466/2012 sobre pesquisa envolvendo seres humanos (BRASIL, 2013).

\section{Resultados}

Foram contabilizadas 1507 lesões autoprovocadas no período estudado, sendo que 1443 pessoas tiveram lesão autoprovocada apenas uma vez e 29 pessoas tiveram mais de uma vez. Das 1472 pessoas com lesão autoprovocada na primeira notificação, 424 foram a óbito, resultando em 1048 pessoas para análise da recorrência de lesão autoprovocada.

Em relação às pessoas que tiveram lesão autoprovocada apenas uma vez, houve maior percentual do sexo feminino $(63,79 \%)$, na faixa etária de 20 a 59 anos $(69,38 \%)$, raça/cor parda $(53,48 \%)$, com mais de oito anos de estudo $(35,82 \%)$, sendo que as lesões autoprovocadas ocorreram predominantemente em região urbana $(85,87 \%)$ e na residência $(83,61 \%)($ Tabela 1$)$. Entre os casos de pessoas com algum tipo de deficiência ou transtorno, houve maior percentual de transtorno mental (8,64\%).

Constatou-se maior frequência de recorrência de lesão autoprovocada na faixa etária de 20 a 59 anos (51,72\%), em pessoas sem companheiro $(55,17 \%)$ e da raça/cor parda $(51,72 \%)$. Verifica-se que 72,41\% das pessoas com recorrência de lesões autoprovocadas eram do sexo feminino e as lesões autoprovocadas ocorreram predominantemente na região urbana $(89,66 \%)$ e na residência $(82,76 \%)$ (Tabela 1). Entre os casos de pessoas com algum tipo de deficiência ou transtorno, houve maior percentual de transtorno mental (17,24\%). Destaca-se que 51,71\% das pessoas com recorrências de lesões autoprovocadas utilizaram como meio a AIM na primeira lesão autoprovocada.

Tabela 1. Características sociodemográficas e clínicas de pessoas com uma lesão autoprovocada e de pessoas com mais de uma lesão autoprovocada ( $\mathrm{n}=1048)$. Mato Grosso, MT, 2015-2017.

\begin{tabular}{lcc}
\hline Variável & Não $(\mathbf{n = 1 0 1 9 )}$ & Mais de uma lesão autoprovocada \\
\hline Faixa etária & $289(28,36 \%)$ & Sim (n=29) \\
10 a 19 & $707(69,38 \%)$ & $14(48,28 \%)$ \\
20 a 59 & $23(2,26 \%)$ & $15(51,72 \%)$ \\
60 ou mais & & $0(0 \%)$ \\
Sexo & $650(63,79 \%)$ & \\
Feminino & $369(36,21 \%)$ & $21(72,41 \%)$ \\
Masculino & & $8(27,59 \%)$ \\
Raça/cor & $8(0,79 \%)$ & $1(3,45 \%)$ \\
Amarela & $302(29,64 \%)$ & $9(31,03 \%)$ \\
Branca & $100(9,81 \%)$ & $3(10,34 \%)$ \\
Ignorado & $12(1,18 \%)$ & $1(3,45 \%)$ \\
Indígena & $545(53,48 \%)$ & $15(51,72 \%)$ \\
Parda & $52(5,10 \%)$ & $0(0 \%)$ \\
Preta & 5 & \\
& &
\end{tabular}




\section{Escolaridade}

Analfabeto

Mais de 8 anos

Situação conjugal/Estado civil

Sem companheiro

Com companheiro

Ignorada

Não se aplica

Possui algum tipo de deficiência/transtorno?

Sim

Não

Ignorado

Tipo de deficiência/transtorno

Deficiência

Deficiência e transtorno

Ignorado

Transtorno

Tipo

Deficiência física

Deficiência intelectual

Deficiência visual

Deficiência auditiva

Transtorno mental

Transtorno de comportamento

Outra deficiência

\section{Zona de ocorrência}

Urbana

Rural

Periurbana

Ignorada

Local da ocorrência

Ignorado

Outro

Residência

Suspeita de uso de álcool

Sim

Não

Ignorado

\section{Orientação sexual}

Heterossexual

$$
14(1,37 \%)
$$

$311(30,52 \%)$

$329(32,29 \%)$

$365(35,82 \%)$

$$
\begin{gathered}
0(0 \%) \\
6(20,69 \%) \\
13(44,83 \%) \\
10(34,48 \%)
\end{gathered}
$$

$343(33,67 \%)$

$16(55,17 \%)$

$187(18,35 \%)$

$3(10,34 \%)$

$484(47,50 \%)$

$5(0,49 \%)$

$10(34,48 \%)$

$0(0 \%)$

$152(14,92 \%)$

$10(34,48 \%)$

$7(24,14 \%)$

$12(41,38 \%)$

$503(49,36 \%)$

$12(1,18 \%)$

$0(0 \%)$

$0(0 \%)$

$1(3,45 \%)$

$9(31,03 \%)$

$113(11,09 \%)$

$5(0,49 \%)$

$0(0 \%)$

$0(0 \%)$

$0(0 \%)$

$0(0 \%)$

$5(17,24 \%)$

$7(24,14 \%)$

$0(0 \%)$

$26(89,66 \%)$

$2(6,90 \%)$

$0(0 \%)$

$1(3,45 \%)$

$54(5,30 \%)$

$55(5,40 \%)$

$2(6,90 \%)$

$3(10,34 \%)$

$24(82,76 \%)$

$852(83,61 \%)$

$118(11,58 \%)$

$2(6,90 \%)$

$304(29,83 \%)$

$10(34,48 \%)$

$597(58,59 \%)$

$17(58,62 \%)$

$369(36,21 \%)$ 


$\begin{array}{lcc}\text { Homossexual } & 20(1,96 \%) & 2(6,90 \%) \\ \text { Não se aplica } & 51(5,00 \%) & 3(10,34 \%) \\ \text { Ignorado } & 579(56,82 \%) & 15(51,72 \%) \\ \text { Situação no mercado de trabalho } & & \\ \text { Ativo } & 142(13,94 \%) & 4(13,79 \%) \\ \text { Inativo } & 100(9,81 \%) & 0(0 \%) \\ \text { Outros } & 73(7,16 \%) & 5(17,24 \%) \\ \text { Ignorado } & 104(69,09 \%) & 20(68,97 \%)\end{array}$

Autointoxicação intencional por medicamento (na primeira lesão autoprovocada)

$\begin{array}{lll}\text { Não } & 653(64,08 \%) & 14(48,28 \%) \\ \text { Sim } & 366(35,92 \%) & 15(51,72 \%)\end{array}$

*A pessoa pode ter mais de uma deficiência e/ou transtorno, por isso a soma dos tipos de deficiência e/ou transtorno não é $100 \%$. Fonte: Autores.

Foi elaborado modelo de regressão logística para análise dos preditores das recorrências de lesões autoprovocadas. Constatou-se que contribuíram de forma significativa para o modelo as variáveis "faixa etária" e "possui algum tipo de deficiência ou transtorno". Houve evidências de maior chance de recorrência de lesão autoprovocada entre pessoas com idade de 10 a 19 anos em comparação às pessoas 20 a 59 anos (OR 2,28) e maior chance de recorrência de lesão autoprovocada entre pessoas que possuem algum tipo de deficiência ou transtorno em comparação às pessoas que não os possuem (OR 1,85). Entre os analfabetos e inativos não houve casos de recorrência, desse modo, foi impossível estimar o OR (Tabela 2). 
Tabela 2. Modelo de regressão logística para análise dos preditores das recorrências de lesões autoprovocadas (n=1048). Mato Grosso, MT, 2015-2017.

\begin{tabular}{|c|c|c|c|c|}
\hline \multirow{2}{*}{$\begin{array}{l}\text { Comparações } \\
\text { Faixa etária (10 a } 19 \text { vs } 20 \text { a 59) }\end{array}$} & \multirow{2}{*}{$\begin{array}{c}\text { Odds Ratio } \\
2,28\end{array}$} & \multirow{2}{*}{\multicolumn{2}{|c|}{$\begin{array}{r}\text { IC 95\% } \\
4,79\end{array}$}} & \multirow{2}{*}{$\begin{array}{c}\begin{array}{c}\text { Valor- } \\
\mathbf{p}\end{array} \\
0,03\end{array}$} \\
\hline & & & & \\
\hline \multicolumn{5}{|l|}{ Faixa etária (10 a 19 vs $60+$ ) } \\
\hline \multicolumn{5}{|l|}{ Faixa etária (20 a 59 vs $60+)$} \\
\hline $\operatorname{Sexo}(\mathrm{F}$ vs M) & 1,49 & 0,65 & 3,40 & 0,34 \\
\hline Raça/Cor (Branca vs Indígena) & 0,36 & 0,04 & 3,05 & 0,35 \\
\hline Raça/Cor (Branca vs Outra) & 0,74 & 0,32 & 1,69 & 0,47 \\
\hline Raça/Cor (Indígena vs Outra) & 2,07 & 0,52 & 8,27 & 0,31 \\
\hline Escolaridade (Analfabeto vs Até 8 anos) & 0,00 & 0,00 & $3,2 \mathrm{E}+221$ & 0,97 \\
\hline Escolaridade (Analfabeto vs Mais de 8) & 0,00 & 0,00 & $4,17 \mathrm{E}+147$ & 0,97 \\
\hline Escolaridade (Até 8 anos vs Mais de 8) & 23,74 & 0,00 & $4,339 \mathrm{E}+76$ & 0,97 \\
\hline Situação conjugal (Com companheiro vs Sem companheiro) & 0,59 & 0,31 & 1,09 & 0,09 \\
\hline Possui algum tipo de deficiência/transtorno? (Sim vs Não) & 1,85 & 1,13 & 3,03 & 0,01 \\
\hline Zona de ocorrência (Urbana vs Não urbana) & 1,16 & 0,56 & 2,39 & 0,70 \\
\hline Local da ocorrência (Residência vs Outro) & 1,03 & 0,56 & 1,88 & 0,94 \\
\hline Suspeita de uso de álcool? (Sim vs Não) & 0,72 & 0,33 & 1,54 & 0,40 \\
\hline Orientação sexual (Heterossexual vs Homossexual) & 0,49 & 0,22 & 1,10 & 0,08 \\
\hline Situação no mercado de trabalho (Inativo vs Ativo) & 0,00 & 0,00 & $1,64 \mathrm{E}+121$ & 0,94 \\
\hline Situação no mercado de trabalho (Inativo vs outra situação) & 0,00 & 0,00 & $4,793 \mathrm{E}+80$ & 0,94 \\
\hline Situação no mercado de trabalho (Ativo vs outra situação) & 29,39 & 0,00 & $2,956 \mathrm{E}+43$ & 0,95 \\
\hline Autointoxicação intencional por medicamento (Sim vs Não) & 1,38 & 0,96 & 2,00 & 0,09 \\
\hline
\end{tabular}

Fonte: Autores.

Observou-se que, das 1472 pessoas com lesão autoprovocada, 428 pessoas (29,07\%) foram a óbito por suicídio (sendo que 424 pessoas foram a óbito na primeira lesão, três pessoas foram a óbito na segunda e uma pessoa foi a óbito na terceira) (Tabela 3)

Na Tabela 3 é possível constatar que, em relação às pessoas que foram a óbito por suicídio, houve maior percentual daquelas do sexo masculino (78,74\%), na faixa etária de 20 a 59 anos (73,36\%), da raça/cor parda (56,54\%), com mais de oito anos de estudo $(62,62 \%)$ e sem companheiro (59,58\%). Ressalta-se a ocorrência de 13 (3,03\%) óbitos por suicídio por AIM, sendo que esses óbitos ocorreram na primeira lesão autoprovocada.

Vale mencionar a frequência elevada de informação "ignorada" para as variáveis investigadas neste estudo. 
Tabela 3. Características sociodemográficas e clínicas de pessoas que não foram à óbito por suicídio e de pessoas que foram a óbito por suicídio (n=1507). Mato Grosso, MT, 2015-2017.

\begin{tabular}{|c|c|c|}
\hline \multirow[t]{2}{*}{ Variável } & \multicolumn{2}{|c|}{ Óbito } \\
\hline & Não & Sim \\
\hline \multicolumn{3}{|l|}{ Faixa etária } \\
\hline 10 a 19 & $320(29,66 \%)$ & $43(10,05 \%)$ \\
\hline 20 a 59 & $736(68,21 \%)$ & $314(73,36 \%)$ \\
\hline 60 ou mais & $23(2,13 \%)$ & $66(15,42 \%)$ \\
\hline Ignorada & $0(0 \%)$ & $5(1,17 \%)$ \\
\hline \multicolumn{3}{|l|}{ Sexo } \\
\hline Feminino & $696(64,50 \%)$ & $90(21,03 \%)$ \\
\hline Masculino & $383(35,50 \%)$ & $337(78,74 \%)$ \\
\hline Ignorado & $0(0 \%)$ & $1(0,23 \%)$ \\
\hline \multicolumn{3}{|l|}{ Raça/cor } \\
\hline Amarela & $9(0,83 \%)$ & $1(0,23 \%)$ \\
\hline Branca & $320(29,66 \%)$ & $146(34,11 \%)$ \\
\hline Indígena & $13(1,2 \%)$ & $2(0,47 \%)$ \\
\hline Parda & $579(53,66 \%)$ & $242(56,54 \%)$ \\
\hline Preta & $52(4,82 \%)$ & $27(6,31 \%)$ \\
\hline Ignorada & $106(9,82 \%)$ & $10(2,34 \%)$ \\
\hline \multicolumn{3}{|l|}{ Escolaridade } \\
\hline Analfabeto & $14(1,3 \%)$ & $17(3,97 \%)$ \\
\hline Até 8 anos & $325(30,12 \%)$ & $104(24,3 \%)$ \\
\hline Mais de 8 anos & $392(36,33 \%)$ & $268(62,62 \%)$ \\
\hline Ignorado & $348(32,25 \%)$ & $39(9,11 \%)$ \\
\hline \multicolumn{3}{|c|}{ Situação conjugal/Estado civil } \\
\hline Com companheiro & $191(17,7 \%)$ & $129(30,14 \%)$ \\
\hline Não se aplica & $5(0,46 \%)$ & $0(0 \%)$ \\
\hline Sem companheiro & $374(34,67 \%)$ & $255(59,58 \%)$ \\
\hline Ignorada & $509(47,17 \%)$ & $44(10,28 \%)$ \\
\hline \multicolumn{3}{|c|}{ Possui algum tipo de deficiência/transtorno? } \\
\hline $\begin{array}{l}\text { Sim } \\
\text { Não }\end{array}$ & $\begin{array}{l}172(15,94 \%) \\
378(35,03 \%)\end{array}$ & $\begin{array}{c}3(0,7 \%) \\
16(3,74 \%)\end{array}$ \\
\hline Ignorado & $529(49,03 \%)$ & $409(95,56 \%)$ \\
\hline \multicolumn{3}{|c|}{ Tipo de deficiência ou transtorno } \\
\hline Deficiência & $12(1,11 \%)$ & $0(0 \%)$ \\
\hline Deficiência e transtorno & $11(1,02 \%)$ & $0(0 \%)$ \\
\hline Transtorno & $131(12,14 \%)$ & $2(0,47 \%)$ \\
\hline Ignorado & $18(1,67 \%)$ & $1(0,23 \%)$ \\
\hline \multicolumn{3}{|c|}{ Tipo de deficiência ou transtorno } \\
\hline Deficiência física & $5(0,46 \%)$ & $0(0 \%)$ \\
\hline
\end{tabular}




\begin{tabular}{|c|c|c|}
\hline Deficiência intelectual & $15(1,39 \%)$ & $0(0 \%)$ \\
\hline Deficiência visual & $4(0,37 \%)$ & $0(0 \%)$ \\
\hline Deficiência auditiva & $4(0,37 \%)$ & $0(0 \%)$ \\
\hline Transtorno mental & $100(9,27 \%)$ & $1(0,23 \%)$ \\
\hline Transtorno de comportamento & $69(6,39 \%)$ & $1(0,23 \%)$ \\
\hline Outra deficiência & $12(1,11 \%)$ & $0(0 \%)$ \\
\hline \multicolumn{3}{|l|}{ Zona de ocorrência } \\
\hline Periurbana & $6(0,56 \%)$ & $0(0 \%)$ \\
\hline Rural & $80(7,41 \%)$ & $5(1,17 \%)$ \\
\hline Urbana & $980(90,82 \%)$ & $35(8,18 \%)$ \\
\hline Ignorado & $13(1,2 \%)$ & $388(90,65 \%)$ \\
\hline \multicolumn{3}{|l|}{ Local da ocorrência } \\
\hline Outro & $116(10,75 \%)$ & $5(1,17 \%)$ \\
\hline Residência & $905(83,87 \%)$ & $34(7,94 \%)$ \\
\hline Ignorado & $58(5,38 \%)$ & $389(90,89 \%)$ \\
\hline \multicolumn{3}{|l|}{ Suspeita de uso de álcool } \\
\hline Sim & $123(11,4 \%)$ & $0(0 \%)$ \\
\hline Não & $324(30,03 \%)$ & $9(2,1 \%)$ \\
\hline Ignorado & $632(58,57 \%)$ & $419(97,9 \%)$ \\
\hline \multicolumn{3}{|l|}{ Orientação sexual } \\
\hline Heterossexual & $393(36,42 \%)$ & $15(3,5 \%)$ \\
\hline Homossexual & $23(2,13 \%)$ & $1(0,23 \%)$ \\
\hline Ignorado & $663(61,44 \%)$ & $412(96,26 \%)$ \\
\hline \multicolumn{3}{|c|}{ Situação no mercado de trabalho } \\
\hline Ativo & $146(13,53 \%)$ & $2(0,47 \%)$ \\
\hline Inativo & $103(9,55 \%)$ & $1(0,23 \%)$ \\
\hline Outros & $85(7,88 \%)$ & $0(0 \%)$ \\
\hline Ignorado & $745(69,05 \%)$ & $425(99,3 \%)$ \\
\hline \multicolumn{3}{|c|}{ Intoxicação exógena por medicamento } \\
\hline Não & $680(63,02 \%)$ & $415(96,96 \%)$ \\
\hline Sim & $399(36,98 \%)$ & $13(3,03 \%)$ \\
\hline
\end{tabular}

*A pessoa pode ter mais de uma deficiência e/ou transtorno, por isso a soma dos tipos de deficiência e/ou transtorno não é $100 \%$. Fonte: Autores.

Foi elaborado modelo de regressão logística para análise dos preditores dos óbitos por suicídio. Observa-se, na análise de OR ajustada, que mantiveram associação significativa as variáveis faixa etária, sexo, escolaridade e AIM (Tabela 4).

Constatou-se menor chance de óbito por suicídio entre pessoas com idade de 10 a 19 anos e de 20 a 59 anos quando comparadas àquelas com 60 anos ou mais (OR 0,06 e 0,23, respectivamente). $\mathrm{O}$ mesmo ocorreu com mulheres quando comparadas aos homens (OR 0,21), escolaridade de até oito anos quando comparada a escolaridade com mais de oito anos (OR 0,23). Observou-se menor chance de óbito por suicídio por AIM (OR 0,17) quando comparada a óbitos por suicídio por outros meios utilizados. 
Tabela 4. Modelo de regressão logística para análise de preditores dos óbitos por suicídio ( $\mathrm{n}=1507)$. Mato Grosso, MT, 2015 2017.

\begin{tabular}{|c|c|c|c|c|c|c|c|c|}
\hline \multirow{3}{*}{$\begin{array}{l}\text { Comparações } \\
\text { Faixa etária (10 a } 19 \text { vs } 20 \text { a 59) }\end{array}$} & \multicolumn{4}{|c|}{ Modelo bruto } & \multicolumn{4}{|c|}{ Modelo ajustado } \\
\hline & \multirow{2}{*}{$\begin{array}{c}\text { Odds } \\
\text { Ratio } \\
0,32\end{array}$} & \multicolumn{2}{|c|}{ IC $95 \%$} & \multirow{2}{*}{$\frac{\text { Valor-p }}{<0,01}$} & \multirow{2}{*}{$\begin{array}{r}\text { Odds } \\
\text { Ratio } \\
0,27\end{array}$} & \multicolumn{2}{|c|}{ IC $95 \%$} & \multirow{2}{*}{$\begin{array}{l}\text { Valor- } \\
\frac{\mathbf{p}}{<0,01}\end{array}$} \\
\hline & & 0,22 & 0,45 & & & 0,16 & 0,47 & \\
\hline Faixa etária (10 a 19 vs $60+$ ) & 0,05 & 0,03 & 0,08 & $<0,01$ & 0,06 & 0,02 & 0,17 & $<0,01$ \\
\hline Faixa etária (20 a 59 vs $60+$ ) & 0,15 & 0,09 & 0,25 & $<0,01$ & 0,23 & 0,10 & 0,54 & $<0,01$ \\
\hline $\operatorname{Sexo}(\mathrm{F}$ vs M) & 0,15 & 0,11 & 0,19 & $<0,01$ & 0,21 & 0,13 & 0,32 & $<0,01$ \\
\hline Raça/Cor (Branca vs Indígena) & 2,96 & 0,61 & 14,34 & 0,17 & 3,09 & 0,38 & 24,97 & 0,25 \\
\hline Raça/Cor (Branca vs Outra) & 1,08 & 0,84 & 1,40 & 0,54 & 0,94 & 0,61 & 1,47 & 0,77 \\
\hline Raça/Cor (Indígena vs Outra) & 0,37 & 0,08 & 1,76 & 0,20 & 0,31 & 0,04 & 2,45 & 0,23 \\
\hline Escolaridade (Analfabeto vs Até 8 anos) & 3,79 & 1,70 & 8,46 & $<0,01$ & 2,17 & 0,52 & 9,01 & 0,25 \\
\hline Escolaridade (Analfabeto vs Mais de 8 ) & 1,77 & 0,81 & 3,89 & 0,14 & 0,50 & 0,12 & 2,05 & 0,30 \\
\hline Escolaridade (Até 8 anos vs Mais de 8) & 0,47 & 0,35 & 0,63 & $<0,01$ & 0,23 & 0,15 & 0,36 & $<0,01$ \\
\hline $\begin{array}{l}\text { Situação conjugal (Com companheiro vs } \\
\text { Sem companheiro) }\end{array}$ & 0,99 & 0,73 & 1,33 & 0,94 & 0,80 & 0,51 & 1,25 & 0,29 \\
\hline $\begin{array}{l}\text { Possui algum tipo de } \\
\text { deficiência/transtorno? (Sim vs Não) }\end{array}$ & 0,41 & 0,10 & 1,63 & 0,19 & & & - & \\
\hline $\begin{array}{l}\text { Tipo de deficiência/transtorno } \\
\text { (Deficiência vs Transtorno) }\end{array}$ & & & & & & & - & \\
\hline $\begin{array}{l}\text { Tipo de deficiência/transtorno } \\
\text { (Deficiência vs Deficiência e Transtorno) }\end{array}$ & & & & & & & - & \\
\hline $\begin{array}{l}\text { Tipo de deficiência/transtorno } \\
\text { (Transtorno vs Deficiência e Transtorno) }\end{array}$ & & & & & & & - & \\
\hline $\begin{array}{l}\text { Zona de ocorrência (Urbana vs Não } \\
\text { urbana) }\end{array}$ & 0,40 & 0,17 & 0,93 & 0,03 & & & - & \\
\hline $\begin{array}{l}\text { Local da ocorrência (Residência vs } \\
\text { Outro) }\end{array}$ & 0,87 & 0,32 & 2,37 & 0,78 & & & - & \\
\hline $\begin{array}{l}\text { Orientação sexual (Heterossexual vs } \\
\text { Homossexual) }\end{array}$ & 0,88 & 0,08 & 10,08 & 0,91 & & & - & \\
\hline $\begin{array}{l}\text { Situação no mercado de trabalho (Inativo } \\
\text { vs Ativo) }\end{array}$ & & & & & & & - & \\
\hline $\begin{array}{l}\text { Situação no mercado de trabalho (Inativo } \\
\text { vs outra situação) }\end{array}$ & & & & & & & - & \\
\hline $\begin{array}{l}\text { Situação no mercado de trabalho (Ativo } \\
\text { vs outra situação) }\end{array}$ & & & & & & & - & \\
\hline Suspeita de uso de álcool? (Sim vs Não) & & & & & & & - & \\
\hline $\begin{array}{l}\text { Autointoxicação intencional por } \\
\text { medicamento (Sim vs Não) }\end{array}$ & 0,05 & 0,03 & 0,10 & $<0,01$ & 0,17 & 0,08 & 0,38 & $<0,01$ \\
\hline $\begin{array}{l}\text { Meio de agressão - Força } \\
\text { corporal/espancamento (Sim vs Não) }\end{array}$ & & & & & & & - & \\
\hline $\begin{array}{l}\text { Meio de agressão - Enforcamento (Sim } \\
\text { vs Não) }\end{array}$ & 12,45 & 5,87 & 26,42 & $<0,01$ & & & - & \\
\hline $\begin{array}{l}\text { Meio de agressão - Objeto contundente } \\
\text { (Sim vs Não) }\end{array}$ & & & & & & & - & \\
\hline $\begin{array}{l}\text { Meio de agressão - Objeto pérfuro- } \\
\text { cortante (Sim vs Não) }\end{array}$ & 0,12 & 0,01 & 0,99 & 0,04 & & & - & \\
\hline $\begin{array}{l}\text { Meio de agressão - Substância/Objeto } \\
\text { quente (Sim vs Não) }\end{array}$ & & & & & & & - & \\
\hline
\end{tabular}




\begin{tabular}{lccccc}
$\begin{array}{l}\text { Meio de agressão - Envenenamento, } \\
\text { intoxicação (Sim vs Não) }\end{array}$ & 0,26 & 0,14 & 0,47 & $<0,01$ & - \\
$\begin{array}{l}\text { Meio de agressão - Arma de fogo (Sim vs } \\
\text { Não) }\end{array}$ & 5,53 & 1,82 & 16,87 & $<0,01$ & - \\
Meio de agressão - Ameaça (Sim vs Não) & & \multicolumn{2}{c}{-} & & - \\
\hline
\end{tabular}

Fonte: Autores.

\section{Discussão}

Nesse estudo foram analisadas 1507 notificações de lesões autoprovocadas, que englobam comportamento suicida e autoagressão, sendo 1443 pessoas com uma lesão autoprovocada. Considerando as características de pessoas com apenas uma lesão autoprovocada notificada, houve predomínio de mulheres, adultos de 20 a 59 anos, de raça/cor parda, com mais de oito anos de escolaridade, que se autolesionaram intencionalmente em residências e na zona urbana.

A literatura aponta como características predominantes das pessoas que tentam suicídio, a idade adulta (Boas, Monteiro, Silva, \& Meneguetti, 2019; Fernandes, Ferreira, \& Castro, 2016; Grigoletto, Souto, Terra, Tisott, \& Ferreira, 2020; Rodrigues, Oliveira, Silva, \& Pinheiro, 2020), sexo feminino, ocorrência em zona urbana e em área domiciliar (Fernandes et al., 2016; Grigoletto et al., 2020; Moreira et al., 2015; Rodrigues et al., 2020) e raça/etnia de cor parda (Fernandes et al., 2016; Rodrigues et al., 2020), corroborando com os resultados da presente pesquisa. No que se refere à escolaridade, estudo revela maior frequência de tentativa de suicídio em pessoas com ensino fundamental incompleto (Rodrigues et al., 2020).

Em relação ao sexo, identificou-se que as mulheres tentam mais suicídio do que os homens (Boas et al., 2019; Baére \& Zanello, 2018; Marquetti \& Marquetti, 2017). Dessa forma, é possível que as mulheres tenham maior vulnerabilidade ao suicídio. São apontados como fatores de risco para suicídio em mulheres, a alta prevalência de transtornos mentais, ciclo menstrual, gravidez; e fatores socioculturais, como o abuso físico, emocional e sexual, além de problemas conjugais. Portanto, programas de prevenção do suicídio devem levar em consideração essas especificidades e incorporarem estratégias específicas voltadas para o sexo feminino (Vijayakumar, 2015).

Destaca-se que a maioria das notificações de lesões autoprovocadas não possuía informações sobre orientação sexual, situação no mercado de trabalho, se possui algum tipo de deficiência ou transtorno e suspeita de uso de álcool. Tais informações também foram ignoradas em grande parte das recorrências de lesões autoprovocadas e óbitos por suicídio. Na maioria das recorrências de lesões autoprovocadas, dados relativos à escolaridade também foram ignorados. Nos óbitos por suicídio, a zona de ocorrência e o local da ocorrência não foram respondidos na maior parte dos casos.

Assim como neste estudo, outras pesquisas apontaram problemas quanto ao preenchimento da notificação de lesões autoprovocadas em razão da inconclusão dos dados (Rosa, Agnolo, Oliveira, Mathias, \& Oliveira, 2016; Rosa, Campos, Guedes, Sales, Mathias, \& Oliveira, 2015; Wieczorkievicz et al., 2016), indicando a necessidade de ações que promovam a notificação adequada dos instrumentos disponibilizados nos serviços de saúde para que seja possível a real compreensão desse agravo na sociedade.

A escassez de informações nos registros merece destaque, pois prejudica a compreensão detalhada sobre a atual situação das lesões autoprovocadas no país. O treinamento e estímulo dos profissionais da saúde voltados à importância da completude desses registros é de extrema relevância, pois dados completos e fidedignos sobre a magnitude da situação permitem a elaboração de políticas públicas específicas voltadas para promoção e prevenção à saúde.

Das 1472 pessoas com lesão autoprovocada na primeira notificação, 29 tiveram mais de uma lesão autoprovocada notificada. O modelo de regressão logística mostrou que as chances de recorrência de lesão autoprovocada foram mais elevadas entre pessoas com idade de 10 a 19 anos em comparação às pessoas de 20 a 59 anos e, também, maior chance de 
recorrência de lesão autoprovocada entre pessoas que possuem algum tipo de deficiência ou transtorno em comparação às pessoas que não os possuem.

No que se refere à faixa etária a literatura sugere maior recorrência entre os jovens (Jeon, Lee, Lee, Hong, Won, Cho, Kim, Chang, Lee, Lee, \& Cho 2010) e adultos jovens (Boas et al., 2019). Nessa perspectiva, estudo referente a dados de jovens (10 a 29 anos) tratados por automutilação na Irlanda, identificou que a recidiva desse ato está relacionada à adolescência nas mulheres (Bennardi, McMahon, Corcoran, Griffin, \& Arensman, 2016).

Embora haja carência de estudos sobre recorrência de lesões autoprovocadas, estudo corrobora os achados da presente pesquisa, ao identificar associação entre recidiva de tentativa de suicídio e presença de história psiquiátrica (Irigoyen-Otiñano, Puigdevall-Ruestes, Mur-Laín, González-Pinto, Portella, Baca-García, \& Lopez-Castroman, 2019). Estudos relatam a associação entre tentativa de suicídio e deficiência/transtorno mental, sendo a depressão a mais frequente (Beitia-Cardona, Rodíguez-Torres, Estrada-González, \& Benavides-Portilla, 2019; Gokcelli, Tasar, Akcam, Sahin, Akarca, Aktas, Duman, Akcicek, \& Noyan, 2017; Jokinen, Talback, Feychting, Ahlbom, \& Ljung, 2018; Pires, Raposo, Sougey, Bastos, Silva, \& Passos, 2015). Jokinen et al. (2018) também apontam que a maioria dos pacientes que tentam suicídio tem um transtorno mental coexistente, como depressão, esquizofrenia, abuso de substâncias ou um distúrbio de personalidade.

Pesquisa que analisou aspectos relacionados às recorrências de tentativas de suicídio em um Departamento de Emergência Hospitalar, no período de um ano após um episódio de comportamento suicida, observou prevalência de recorrência em 23,1\% da amostra, estando entre os preditores para a recorrência, maior número de tentativa de suicídio anterior (Suárez-Pinilla, Pérez-Herrera, Suárez-Pinilla, Medina-Blanco, López-García, Artral-Simón, \& Santiago-Díaz, 2020).

Em relação aos óbitos por suicídio, o modelo de regressão logística revelou menor chance de óbito entre pessoas mais jovens, mulheres, com até oito anos de escolaridade e com AIM como método utilizado.

Quanto à idade, em consonância com a presente pesquisa, estudos revelam predominância de óbito por suicídio em idosos (Baére \& Zanello, 2018; Santos, 2010). Vedana (2018) menciona que o aumento da expectativa de vida em diferentes contextos nem sempre vem acompanhada por iniciativas de impacto voltadas para a saúde mental ou promoção da qualidade de vida do idoso. Aponta a necessidade de pesquisas e planos de ação direcionados ao cuidado integral ao idoso que incluam a prevenção dos comportamentos suicidários.

No que se refere à escolaridade, a literatura revela maior frequência de óbitos por suicídio em indivíduos com baixa escolaridade (Felix, Oliveira, Campos, Dias, Parente, Freire, Ferreira, Lima, \& Ximenes, 2020; Galvão, Ribeiro, \& Nery, 2019; Parente, Flor, Alves, Dias, Brito, \& Vasconselos, 2016). O baixo nível de escolaridade geralmente está vinculado a dificuldades socioeconômicas, com grandes prejuízos à qualidade de vida individual e familiar, com consequente aumento do risco de comportamentos suicidas (Seleghim, Bellasalma, Mathias, \& Oliveira, 2012).

Estudos nacionais e internacionais corroboram os achados da presente pesquisa no que se refere a menor frequência de óbito por suicídio em mulheres quando comparadas aos homens (Carmo Santos, Ribeiro, Soares, Santana, Bomfim, Oliveira, \& Oliveira, 2018; Chen et al., 2016; Cuchara \& Diaz, 2020; Dendup, Zhao, Dorji, \& Phuntsho, 2020; Ribeiro, Castro, Scatena, \& Haas, 2018; Sagna, Kemp, DiNittio, \& Choi, 2020). Considerando o maior percentual de óbito por suicídio entre pessoas do sexo masculino e a menor chance de óbito por suicídio entre mulheres, é possível indicar a utilização de métodos de baixa letalidade entre as mulheres e de alta letalidade entre os homens (Felix et al., 2020; Santos, Oliveira, Azevedo, Nunes, Amador, \& Barbosa, 2017).

Pesquisa realizada em Navarra (Espanha) identificou que os três métodos mais utilizados para cometer suicídio foram enforcamento (32,8\%), precipitação em altura $(22,8 \%)$ e excesso de ingestão farmacológica (12,8\%). Entretanto, foi encontrada diferença em relação à opção escolhida por homens e mulheres. Os métodos mais utilizados pelos homens foram enforcamento (34,6\%), precipitação em altura $(24,3 \%)$ e armas de fogo $(11,0 \%)$. Entre as mulheres, foram enforcamento 
$(27,3 \%)$ e excesso de ingestão farmacológica (27,3\%), seguidos pela precipitação da altura (18,2\%) (Azcárate et al., 2015), sustentando a hipótese dessa população utilizar métodos menos violentos se comparada aos homens.

Na presente pesquisa, houve menor chance de óbito por suicídio quando o método utilizado foi a AIM. Tal aspecto merece ser investigado em outros estudos, pois considerando essa informação, é possível verificar que a letalidade associada aos medicamentos tenha sido menor quando comparada à de outros grupos de agentes tóxicos ou demais métodos utilizados, porém, ressalta-se nesse estudo, a ocorrência dos 13 (3,03\%) óbitos por suicídio por AIM na primeira lesão autoprovocada.

Estudos que avaliaram o padrão de intoxicação entre pacientes em centros de atendimento terciário, constataram que a intoxicação por comprimidos, principalmente benzodiazepínicos foi predominante (Fernández-Ibáñes, Ugalde-Herrá, Rodriguez-Getino, Garcia-Casas, \& Diaz-Suarez, 2020; Suganthi \& Raja, 2019). Dentre os fatores de risco, merecem destaque as mulheres, pois tentam mais suicídio com uso de medicamentos (Junior, Santos, Silva, Gomes, \& Ribeiro, 2019) quando comparadas aos homens (Azcárate-Juménez et al., 2019; Bochner \& Freire, 2018; Rodrigues et al., 2020).

Pesquisa revelou que os agentes tóxicos mais usados nas tentativas de suicídio foram os praguicidas $(42,9 \%)$ seguidos pelos medicamentos (39,5\%). A maioria dos indivíduos eram mulheres, com idades de 12 a 39 anos e que tentaram suicídio em suas residências (Gondim, Nogueira, Lima, Lima, Albuquerque, Veras, \& Ferreira, 2017). No ambiente residencial é frequente o acesso e a disponibilidade de agentes tóxicos para o próprio uso da vítima (Moreira et al., 2015), merecendo ser considerado para elaboração de planos de gestão de crises.

\section{Considerações Finais}

Os achados desse estudo revelaram variáveis sociodemográficas e clínicas que constituem preditores para recorrência de lesões autoprovocadas e óbitos por suicídio, fornecendo embasamento científico para a implementação de ações por profissionais de saúde e contribuindo com importantes subsídios para programas de proteção e prevenção ao suicídio.

Os resultados da presente pesquisa devem ser interpretados considerando-se suas limitações. A primeira refere-se à impossibilidade de estabelecer as relações de causa e efeito entre as variáveis, dada à sua natureza de coorte transversal. Outra limitação refere-se ao fato da amostra em estudo ser limitada a um curto período de tempo; por abordar dados secundários de sistemas de informação em saúde com escassez de informações e percentual elevado de opções "ignoradas”.

As Fichas de notificação adotadas neste estudo são amplamente utilizadas em pesquisas no território nacional e para notificação compulsória. Constata-se a necessidade de ações voltadas à completude do registro nos instrumentos disponibilizados nos serviços de saúde, para que seja possível a real compreensão desse agravo e a elaboração de políticas públicas específicas visando a prevenção de lesões autoprovocadas e óbitos por suicídio. Desse modo, esse estudo reforça a importância de ações para melhorar as notificações de lesões autoprovocadas, bem como o registro dos óbitos por suicídio, para garantir o acesso a dados confiáveis e sem lacunas de informação.

Os achados apontam, ainda, a necessidade de avanço acerca do conhecimento relacionado ao comportamento suicida, a importância do envolvimento de gestores e profissionais de saúde no planejamento de ações integradas em saúde e na definição de intervenções voltadas à prevenção

Além disso, estudos longitudinais devem ser conduzidos para ampliar o conhecimento acerca das características e dos preditores para recorrência de lesões autoprovocadas e óbitos por suicídio, auxiliando no desenvolvimento e aperfeiçoamento de políticas públicas acerca do tema 


\section{Agradecimentos}

A Coordenação de Aperfeiçoamente de Pessoal de Nível Superior - Brasil (CAPES) - pelo apoio financeiro. MIASSO, A.I. é Bolsista de Produtividade em Pesquisa e agradece ao Conselho Nacional de Desenvolvimento Científico e Tecnológico (CNPq) o apoio recebido para o desenvolvimento de estudos sobre o tema.

\section{Referências}

Azcárate, L., Peinado, R., Blanco, M., Goñi, A., Cuesta, M. J., Prandini, I., \& López-Goñi, J. J. (2015). Características de los suicidios consumados en Navarra en función del sexo (2010-2013). An. Sist. Sanit. Navar, 38(1):9-20.

Azcárate-Juménez, L., Goñi, J. J. L., Goñi-Sarriés, A., Reula-Montes, L., Fernández-Portilla, A., \& Elorza-Pardo, R. (2019). Repeated suicide attempts: a follow-up study. Actas Esp Psiquiatri, 47(4):127-136.

Baére, F., \& Zanello, V. (2018). O gênero no comportamento suicida: uma leitura epidemiológica dos dados do Distrito Federal. Estudos de Psicologia, 23(2):168-178.

Bahia, C. A., Avanci, J. Q., Pinto, L. W., \& Minayo, M. C. S. (2020) Notificações e internações por lesões autoprovocadas em adolescentes no Brasil, 20072016. Epidemiol. Serv. Saude, 29(2):e2019060.

Beitia-Cardona, P., Rodíguez-Torres, E., Estrada-González, C., \& Benavides-Portilla, M. (2019). Suicide attempts and associated factors in two institutions of Cali - Colombia. Revista Ciencia y/ Cuidado, 16(1):19-31

Bennardi, M., McMahon, E., Corcoran, P., Griffin, E., \& Arensman, E. (2016). Risk of repeated self-harm and associated factors in children, adolescents and young adults. BMC Psychiatry,16(1):42.

Boas, A. C. V., Monteiro, Q. R. S., Silva, R. P. M., \& Meneguetti, D. U. O. (2019). Perfil das Tentativas de Suicídio Atendidas em um Hospital Público de Rio Branco, Acre de 2007 a 2016. J Hum Growth, 29(1): 57-64.

Bochner, R., \& Freire, M. M. (2018). Análise dos óbitos decorrentes de intoxicação ocorridos no Brasil de 2010 a 2015 com base no Sistema de Informação sobre Mortalidade (SIM). Cien Saude Colet, 25(2):761-772.

Brasil. (2013). Ministério da Saúde. Conselho Nacional de Saúde. Resolução nº 466, de 12 de dezembro de 2012 . Aprovar as seguintes diretrizes e normas regulamentadoras de pesquisas envolvendo seres humanos. Diário Oficial da União, Brasília, DF, Seção 1.

Brasil. (2019). Ministério da Saúde (MS). Secretaria de Vigilância em Saúde. Departamento de Doenças e Agravos não Transmissíveis e Promoção da Saúde. Saúde Brasil 2018 uma análise da situação de saúde e das doenças e agravos crônicos: desafios e perspectivas. Brasília: MS.

Calixto Filho, M., \& Zerbini, T. (2016). Epidemiologia do suicídio no Brasil entre os anos de 2000 e 2010. Saúde, Ética \& Justiça, 21 (2):45-51.

Camargo Junior, K. R., \& Coeli, C. M. (2000). Reclink: aplicativo para o relacionamento de bases de dados, implementando o método probabilistic record linkage. Cad Saude Colet, 16(2):439-447.

Carmo, E. A., Santos, P. H. S., Ribeiro, B. S., Soares, C. J., Santana, M. L. A. D., Bomfim, E. S., Oliveira, B. G., \& Oliveira, J. S. (2018). Sociodemographic characteristics and time series of mortality due to suicide among elderly individuals in Bahia State, Brazil, 1996-2013. Epidemiol Serv Saude, 27 (1): 1-8.

Chen, I. M., Liao, S. C., Lee, M. G., Wu, C. Y., Lin, P. H., \& Chen, W. J. (2016). Risk factors of suicide mortality among multiple attempters: A national registry study in Taiwan. J Formos Med Assoc, 115(5):364-371.

Cicogna, J. I. R., Hillesheim, D., \& Hallal, A. L. L. (2019). Mortalidade por suicídio de adolescentes no Brasil: tendência temporal de crescimento entre 2000 e 2015. J Bras Psiquiatr, 68(1):1-7.

Conejero, I., Castroman-Lopez, J., Giner, L., Baca-Garcia, E. (2016). Sociodemographic Antecedent Validators of Suicidal Behavior: A Review of Recent Literature. Curr Psychiatry Rep, 18:94.

Cuchara, B., \& Diaz, F. J. (2020). An 8-Year Retrospective Study on Suicides in Washington, DC. Am J Forensic Med Pathol, 41(1):18-26.

Dendup, T., Zhao, Y., Dorji, T., \& Phuntsho, S. (2020). Risk factors associated with suicidal ideation and suicide attempts in Bhutan: An analysis of the 2014 Bhutan STEPS Survey data. PloS One, 15(1):1-13.

Felix, T. A., Oliveira, E. N., Campos, M. P., Dias, M. A. S., Parente, J. R. F., Freire, M. A., Ferreira, F. V., Lima, G. F., \& Ximenes F. R. G. N. (2020) Alguma coisa está fora da ordem: características de casos de tentativa de suicídio atendidos em uma emergência de hospital de grande porte. Research, Society and Development, 9(8):e792986361.

Fernandes, D. A. A., Ferreira, N. S., \& Castro, J. G. D. (2016) Perfil epidemiológico das tentativas de suicídio em Palmas-Tocantins, de 2010 a 2014. Tempus, actas de saúde colet, Brasília, 10(4):09-23.

Fernández-Ibáñes, A., Ugalde-Herrá, R., Rodriguez-Getino, J. A., Garcia-Casas, J. B., \& Diaz-Suarez, J. C. (2020) Epidemiología de las intoxicaciones agudas por sustancias de abuso en Urgencias. Estudio descriptivo en el área IV de Asturias. Adicciones, 20(10):20-20.

Ferreira, T. D. G., Vedana, K. G. G., Amaral, L. C., Pereira, C. C. M., Zanetti, A. C. G., Miasso, A. I., \& Borges, T. L. (20 19). Assistance related to suicidal behavior at a mobile emergency service: Sociodemographic and clinical associated factors. Arch Psychiatr Nurs, 33(2):136-142.

Finkelstein, Y., Macdonald, E. M., Hollands, S., Sivilotti, M. L. A., Hutson, J. R., Mamdani, M. M., Koren, G., \& Juurlink, D. N. (2015). Risk of suicide following deliberate self-poisoning. JAMA Psychiatry, 72(6):570-575. 
Fond, G., Llorca, P. M., Boucekine, M., Zendjidjian, X., Brunel, L., Lancon, C., Auquier, P., \& Boyer, L. (2016). Disparities in suicide mortality trends between United States of America and 25 European countries: retrospective analysis of WHO mortality database. Scientific Reports, 6:20256.

Galvão, C. V. T., Ribeiro, D. L. N., \& Nery, F. S. (2019). Caracterização do suicídio segundo ocupação no estado de Sergipe. Ciências Biológicas e de Saúde, $5(3): 13-26$

Gokcelli, D. K., Tasar, P. T., Akcam, N. O., Sahin, S., Akarca, F. K., Aktas, E. O., Duman, S., Akcicek, F., \& Noyan, A. (2017). Evaluation of attempted older adults suicides admitted to a University Hospital Emergency Department: Izmir study. Asian Journal of Psychiatry, 30:196-199.

Gondim, A. P. S., Nogueira, R. R., Lima, J. G. B., Lima, R. A. C., Albuquerque, P. L. M. M., Veras, M. S. B., \& Ferreira, M. A. D. (2017). Tentativas de suicídio por exposição a agentes tóxicos registradas em um Centro de Informação e Assistência Toxicológica em Fortaleza, Ceará, 2013. Epidemiol. Serv. Saude, 26(1):109-119.

Grigoletto, A. P., Souto, V. T., Terra, M. G., Tisott, Z. L., \& Ferreira, C. N. (2020). Suicide attempts notified in a teaching hospital in the state of Rio Grande do Sul, 2014-2016. Revista de Pesquisa: Cuidado é Fundamental (Online), 12:413-419.

Haghparast-Bidgoli, H., Rinaldi, G., Shahnavazi, H., Bouraghi, H., \& Kiadaliri, A. A. (2018). Socio-demographic and economics factors associated with suicide mortality in Iran, 2001-2010: application of a decomposition model. Int j equity health, 17(1):77.

India State-Level Disease Burden Initiative Suicide Collaborators. (2018). Gender differentials and state variations in suicide deaths in India: the Global Burden of Disease Study 1990-2016. Lancet Global Health, 3: 478-489.

Irigoyen-Otiñano, M. I., Puigdevall-Ruestes, M., Mur-Laín, M., González-Pinto, A., Portella, M. J., Baca-García, E., \& Lopez-Castroman, J. (2019). Ausencia de asociación entre el nivel de letalidad y la reincidencia de los intentos de suicidio en una provincia española. Actas Esp Psiquiatr, 47(5):179-189.

Jaro, M. A. (1995). Probabilistic linkage of large public health data files. Stat Med, 14(5-7):491-498.

Jeon, H. J., Lee, J. Y., Lee, Y. M., Hong, J. P., Won, S. H., Cho, S. J., Kim, J. Y., Chang, S. M., Lee, D., Lee, H. W., \& Cho, M. J. (2010). Lifetime prevalence and correlates of suicidal ideation, plan, and single and multiple attempts in a Korean nationwide study. The Journal of nervous and mental disease, 198(9), $643-646$.

Jokinen, J., Talback, M., Feychting, M., Ahlbom, A., \& Ljung, R. (2018). Life expectancy after the first suicide attempt. Acta Psychiatr Scand, 137:287-295.

Junior, C. J. S., Santos, I. V., Silva, J. V. S., Gomes, V. M., \& Ribeiro, M. C. (2019). Perfil de pacientes atendidos por tentativa de suicídio em um Hospital Geral de Emergências do estado de Alagoas, Brasil. Medicina (Ribeirao Preto Online) [Internet], 52(3):223-30.

Machado, D. B., \& Santos, D. N. (2015) Suicídio no Brasil, de 2000 a 2012. J Bras Psiquiatr, 64(1):45-54.

Marquett,i F. R., \& Marquetti, F. C. (2017). Sicídio e Feminilidades. Cardernos pagu (49), e174921

Moreira, D. L., Martins, M. C., Gubert, F. A., \& Sousa, F. S. P. (2015). Perfil de los pacientes tratados por intento de suicidio en un centro de atención toxicológica. Ciencia y Enfermaria XXI, 21(2):63-75.

Naghavi, M. (2019). Global Burden of Disease Self-Harm Collaborators Global, Regional, and National Burden of Suicide Mortality 1990 to 2016: Systematic Analysis for the Global Burden of Disease Study 2016. BMJ, 326: 169.

Oliveira, L. R., \& Benedetti, A. O. C. (2018). Suicídio em Mato Grosso - Brasil: 1996 a 2015. J. Health Biol Sci., 6(4):391-398.

Omezzine, R. G. (2019). Psychiatric Emergencies: Factors Associated With Suicide Attempts. La tunisie medicale, 97(7):910-917

Parente, A. C., Flor, S. M. C., Alves, V. J., Dias, M. A. S., Brito, M. C. C., \& Vasconselos, F. J. L. (2016). Perfil dos cas os de suicídio em Sobral entre os anos de 2010 e 2015. Sanare, 15(2): 15-22

Parra-Uribe, I., Blasco-Fontecilla, H., Garcia-Parés, G., Martínez-Naval, L., Valero-Coppin, O., Cebrià-Meca, A., Oquendo, M. A., \& Palao-Vidal, D. (2017). Risk of re-attempts and suicide death after a suicide attempt: A survival analysis. BMC Psychiatry, 17:163.

Pereira, A.S., Shitsuka, D. M., Parreira, F. J., \& Shitsuka, R. (2018). Metodologia da pesquisa científica. Ed. UAB/NTE/UFSM.

Pires, Maria Cláudia da Cruz, Raposo, Maria Cristina Falcão, Sougey, Everton Botelho, Bastos Filho, Othon Coelho, Silva, Tatiana Santana, \& Passos, Marcela Pires dos. (2015). Indicadores de risco para tentativa de suicídio por envenenamento: um estudo caso-controle. Jornal Brasileiro de Psiquiatria, 64(3), 193-199

Ribeiro, N. M., Castro, S. D. E., Scatena, L. M., \& Haas, V. J. (2018). Análise da Tendência temporal do suicídio e de Sistemas de Informações em Saúde em Relação às Tentativas de Suicídio. Texto Contexto Enferm, 27(2):e2110016.

Rodrigues, M. F., Oliveira, P. P., Silva, H. C., \& Pinheiro, J. M. C. (2020). Comportamento suicida: o perfil epidemiológico das lesões autoprovocadas no estado de Goiás. Rev Cient Esc Estadual Saúde Pública Goiás "Cândido Santiago", 6(2):e20001.

Rosa, N. M., Agnolo, C. M. D., Oliveira, R. R., Mathias, T. A. F., \& Oliveira, M. L. F. (2016). Tentativas de suicídio e suicídios na atenção pré-hospitalar. J Bras Psiquiatr, 65(3):231-238.

Rosa, N. M., Campos, A. P. S., Guedes, M. R. J., Sales, C. C. F., Mathias, T. A. F., \& Oliveira, M. L. F. (2015). Intoxicações associadas às tentativas de suicídio e suicídio em crianças e adolescentes. Rev enferm UFPE on line, 9(2):661-668.

Sagna, A. O., Kemp, M. L. S., DiNittio, D. M., \& Choi, N. G. (2020) Impact of suicide mortality on life expectancy in the United States, 2011 and 2015: age and sex decomposition. Public Health, 179, 76-89. 
Research, Society and Development, v. 10, n. 2, e4110212142, 2021

(CC BY 4.0) | ISSN 2525-3409 | DOI: http://dx.doi.org/10.33448/rsd-v10i2.12142

Santos, E. G. O., Oliveira, Y. O. M. C. O., Azevedo, U. M., Nunes, A. D. S., Amador, A. E., \& Barbosa, I. R. (2017). Análise espaço-temporal da mortalidade por suicídio em idosos no Brasil. Rev Bras Geriatr. Gerontol. 20(6):854-65.

Santos, J. (2010). Suicídio em Mato Grosso do Sul, Brasil: fatores sociodemográficos [dissertação]. Brasil: Escola Nacional de Saúde Púlica Sergio Auroca (ENSP).

Seleghim, M. R., Bellasalma, A. C. M., Mathias, T. A. F., \& Oliveira, M. L. F. (2012) Caracterização das tentativas de suicídio entre idosos. Cogitare Enferm., $17(2): 277-83$.

Suárez-Pinilla, P., Pérez-Herrera, M., Suárez-Pinilla, M., Medina-Blanco, R., López-García, E., Artral-Simón, J. A., \& Santiago-Díaz, A. I. (2020) Recurrence of suicidal thoughts and behaviors during one year of follow-up: An exploratory study. Psychiatry Res, 1-10.

Suganthi, S., \& Raja, V. P. (2019) Poisoning and its pattern among patients in a tertiary care center in Kancheepuram district, Tamil Nadu. J Edu Health Promo, 8:194.

Vedana, K. G. G. (2018). Iniciativas estratégicas para a prevenção dos comportamentos suicidários em idosos. Revista de Enfermagem Referência, 4(ed16):3-6

Vieira, L. P., Santana, V. T. P., \& Suchara, E. A. (2015). Characterization of suicide attempts by exogenous substances. Cad Saude Colet, 23(2):118-123.

Vijayakumar, L. (2015). Suicide in women. Indian J Psychiatry, 57(6):233-238.

Wieczorkievicz, A. M., Mazon, L. M., Raissa, C. M., Maia, E. W., \& Siebeneichler, J. L. (2016). Saúde Ver, 16(43):53-62.

World Health Organization (WHO). (2014). Preventing suicide: a global imperative. Geneva: WHO.

World Health Organization (WHO). (2002). World report on violence and health. Geneva: WHO. 